\title{
Identificación de Mycobacterium bovis en pacientes con diagnóstico de tuberculosis pulmonar y extrapulmonar
}

\author{
Alejandro Hernández-Solís, ${ }^{1 *}$ Maribel González-Villa, ${ }^{2}$ Raúl Cícero-Sabido, ${ }^{1}$ \\ Heleodora González-González, ${ }^{1}$ Yesenia Colín-Muñoz, ${ }^{1}$ Alejandra Camerino-Guerrero ${ }^{1}$ y \\ Ernesto Ramírez-González² \\ 'Secretaría de Salud, Hospital General de México "Dr. Eduardo Liceaga", Unidad de Neumología; 'Secretaría de Salud, Instituto de Diagnóstico y \\ Referencia Epidemiológicos. Ciudad de México, México
}

\section{Resumen}

Introducción: En México existe un incremento alarmante de casos de infección pulmonar y extrapulmonar por Mycobacterium bovis. La falta de identificación oportuna deriva en complicaciones y eleva la mortalidad. Objetivo: Conocer la frecuencia de infecciones por Mycobacterium bovis en muestras clínicas de pacientes con tuberculosis, identificadas en el laboratorio de micobacterias en un hospital de concentración de la Ciudad de México. Método: Estudio prospectivo, descriptivo. Se estudiaron cepas aisladas de material biológico en cultivos Löwestein-Jensen y MGITI960. La identificación de Mycobacterium bovis se realizó mediante la amplificación del fragmento RD9 por PCR punto final. Resultados: Se incluyeron 850 pacientes con diagnóstico de tuberculosis, en 441 casos se confirmó Mycobacterium tuberculosis por cultivo positivo (250 casos pulmonares, 65 ganglionares, 39 renales, 34 meníngeos, 25 miliares, 14 pleurales, ocho peritoneales, cuatro óseos y dos pericárdicos). Se tipificaron 48 cepas (10.8 \%) como Mycobacterium bovis por amplificación del fragmento RD9 por PCR punto final. Conclusiones: Actualmente no se piensa en Mycobacterium bovis como agente causal de tuberculosis, 10 que pudiera ser la causa del fracaso del tratamiento farmacológico. En este estudio se observó que la principal forma extrapulmonar es la linfadenopatía cervical.

PALABRAS CLAVE: Tuberculosis. Mycobacterium bovis. PCR punto final.

\begin{abstract}
Introduction: In Mexico, there is an alarming increase in the number of cases of Mycobacterium bovis infection on pulmonary and extrapulmonary presentations. The lack of timely identification triggers complications and increases mortality. Objective: To know the frequency of $M$. bovis infections in clinical samples of patients with tuberculosis in the mycobacteria laboratory of a reference hospital in Mexico City. Method: Prospective, descriptive study. Strains isolated from biological material were studied in Löwestein-Jensen and MGITI960 cultures. M. bovis was identified by amplifying the RD9 fragment with end-point polymerase chain reaction (PCR). Results: Eight-hundred and fitty tuberculosis-diagnosed patients were included; in 441 cases, Mycobacterium tuberculosis was confirmed by positive culture (250 pulmonary, 65 ganglionic, 39 renal, 34 meningeal, 25 miliary, 14 pleural, 8 peritoneal, 4 bone and 2 pericardial cases). Forty-eight strains (10.8\%) were typified as M. bovis by amplification of the RD9 fragment with end-point PCR. Conclusions: M. bovis is not currently thought of a causative agent of tuberculosis, which could be the cause of pharmacological treatment failure. In this study, the main extrapulmonary form was observed to be cervical lymphadenopathy.
\end{abstract}

KEY WORDS: Tuberculosis. Mycobacterium bovis. End-point polymerase chain reaction.

Correspondencia:

*Alejandro Hernández-Solís

E-mail: drhernandezsolis@yahoo.com.mx
Fecha de recepción: 17-07-2019

Fecha de aceptación: 05-09-2019

DOI: 10.24875/GMM.19005407
Gac Med Mex. 2019;155:608-612

Disponible en PubMed www.gacetamedicademexico.com 


\section{Introducción}

Mycobacterium tuberculosis es el principal agente causal de la tuberculosis en humanos y Mycobacterium bovis comúnmente causa tuberculosis en animales. ${ }^{1}$ El término tuberculosis zoonótica se utiliza para describir la infección por Mycobacterium bovis en humanos.

La transmisión de la tuberculosis causada por ambos patógenos puede ocurrir de humanos a animales y viceversa. ${ }^{2}$ La Organización Mundial de la Salud señala que actualmente es un problema de salud pública poco atendido en los países emergentes. En estudios recientes se ha observado coinfección de Mycobacterium bovis y Mycobacterium tuberculosis. ${ }^{3}$

La infección es adquirida por contacto directo e indirecto con animales y sus secreciones, lo que aumenta el riesgo de tuberculosis zoonótica. Los factores de riesgo conocidos son antecedentes laborales del individuo, consumo de leche cruda y productos derivados de la misma, especialmente en las tuberculosis extrapulmonares. ${ }^{3,4}$

La genotipificación de Mycobacterium bovis se ha realizado principalmente mediante patrones moleculares (espoligotipos), sumamente útiles para conocer la propagación de la enfermedad; desafortunadamente es un estudio costoso y no está disponible en todos los laboratorios del país. ${ }^{5}$

En México, el control de esta enfermedad se lleva a cabo mediante el Programa Nacional contra la Tuberculosis Bovina, que ha logrado disminuir la prevalencia de la enfermedad en la mayor parte del país, excepto en las regiones de alta producción lechera. ${ }^{6}$

Las infecciones pulmonares y las presentaciones extrapulmonares no son documentadas ni registradas adecuadamente; se cree que son responsables de 5 a $10 \%$ de todas las infecciones tuberculosas. No todos los individuos infectados por Mycobacterium bovis desarrollan enfermedad activa, si bien los pacientes con inmunodepresión son más propensos a infección. ${ }^{5,6}$

Uno de los retos que enfrenta el personal de salud estriba en identificar la causa de la enfermedad, dado que Mycobacterium bovis y Mycobacterium tuberculosis son clínica y radiológicamente indistinguibles entre sí. Se sospecha el primer caso cuando un paciente no mejora con el tratamiento de primera línea, lo que hace indispensable conocer la especie involucrada para establecer el tratamiento específico ya que Mycobacterium bovis es naturalmente resistente a la pirazinamida. $^{7}$
El objetivo de este estudio es determinar la frecuencia de Mycobacterium bovis en muestras clínicas de pacientes con tuberculosis pulmonar y extrapulmonar, procesadas en el laboratorio de micobacterias de un hospital de tercer nivel de especialidades, de enero de 2013 a diciembre de 2018.

\section{Método}

Se diseñó un estudio prospectivo de muestras clínicas de pacientes que ingresaron con diagnóstico de tuberculosis entre enero de 2013 y diciembre de 2018, para establecer la frecuencia de Mycobacterium bovis como agente etiológico de pacientes portadores de tuberculosis pulmonar y formas extrapulmonares en el Hospital General de México "Dr. Eduardo Liceaga". Los criterios de inclusión fueron pacientes mayores de 18 años en cuyas muestras clínicas (esputo, material de biopsia, orina, líquido pleural y líquido cefalorraquídeo) se obtuviera crecimiento de micobacterias en el medio de cultivo Löwestein-Jensen y MGITI960, sin diagnóstico previo de tuberculosis ni en tratamiento con fármacos antituberculosis.

Al ingreso hospitalario, a cada paciente con diagnóstico de tuberculosis se le realizó historia clínica, citometría hemática, química sanguínea, identificación del virus de inmunodeficiencia humana por ELISA y su confirmación por Western-blot y radiografía de tórax.

En las presentaciones extrapulmonares se practicó biopsia (linfadenopatía cervical, peritoneo, pleura y tejido óseo). Las muestras obtenidas fueron fijadas en formol a $10 \%$ e incluidas en parafina, seccionadas y teñidas con hematoxilina-eosina para examen microscópico. Se realizó el diagnóstico de tuberculosis cuando los hallazgos fueron formación de granulomas con focos de necrosis caseosa, fibrosis y presencia de células epitelioides.

Se realizó toma de material de biopsia con técnica aséptica y cultivo para micobacterias con el medio Löwestein-Jensen y MGTI960, estándar de oro para el diagnóstico de tuberculosis.

En cada muestra se realizó tinción de Ziehl-Neelsen para BAAR, adenosin deaminasa (ADA), reacción en cadena de la polimerasa en punto final (PCR) para la detección de la secuencia de inserción $1 S 6110$ presente en micobacterias del complejo de Mycobacterium tuberculosis y una prueba cutánea con el derivado purificado de la proteína (PPD-RT23, Statens Seruminstitut, Copenhague, Dinamarca).

Los especímenes fueron homogenizados en solución salina isotónica, buffer-fosfato y descontaminados 
con solución de hidróxido de sodio a $4 \%$. Después de la neutralización y la centrifugación, cada homogenizado fue suspendido de nuevo en el agua destilada. Los bacilos ácido-alcohol resistentes (BAAR) fueron detectados con tinción de Ziehl-Neelsen. Para los cultivos, $0.2 \mathrm{~mL}$ de los homogenizados de las muestras fueron inoculados en botellas con el medio líquido fluorescente de MGIT 960 (Tubo del Indicador del Crecimiento de Mycobacterium, Becton Dickinson, San José, CA, Estados Unidos) y medio sólido Löwestein-Jensen. Los cultivos fueron incubados a $37^{\circ} \mathrm{C}$ por ocho semanas e inspeccionados semanalmente para valorar el crecimiento.

El ADN se extrajo por choque térmico de una suspensión bacteriana de cada aislado y se usó como objetivo para la amplificación de aproximadamente 124 pb del producto correspondiente a una secuencia de inserción IS6110 característica del complejo Mycobacterium tuberculosis. Los cebadores de PCR IS6110F (5'-GGATCCGCCAGCCCAGGATCCTGCG-3') e IS6110R (5'-AGGTGCGGACCACCAGCACCTAACC-3') se diseñaron en el Instituto de Diagnóstico y Referencia Epidemiológicos. La mezcla de PCR (50 $\mu \mathrm{L})$ fue $10 \mathrm{mM}$ de Tris- $\mathrm{HCl}$, $1.5 \mathrm{mM}$ de cloruro de magnesio, $50 \mathrm{mM}$ de cloruro de potasio ( $\mathrm{pH}$ 8.3), $1 \mathrm{U}$ de taqpolimerasa, $2 \mathrm{mM}$ de una mezcla de dNTP (Roche Diagnostics GmbH, Mannheim, Alemania), $0.2 \mu \mathrm{M} \mathrm{L}$ de ADN de cada cebador. Las condiciones de reacción fueron las siguientes: un ciclo de un minuto a $94{ }^{\circ} \mathrm{C}$, seguido de 45 ciclos de 15 segundos a $94{ }^{\circ} \mathrm{C}, 50$ segundos a $70{ }^{\circ} \mathrm{C}$ y un ciclo de siete minutos a $72{ }^{\circ} \mathrm{C}$.

Para la identificación molecular de Mycobacterium bovis, la preparación del ADN se realizó mediante el método de ebullición y se usó como plantilla para la PCR genérica del complejo de Mycobacterium. Para diferenciar entre Mycobacterium tuberculosis y Mycobacterium bovis, todas las cepas reportadas como positivas al complejo de micobacterias por PCR fueron analizadas por un sistema de PCR de punto final RD9 de tres cebadores (RD9F, RD9R y RD9I), método zs|2ew por Samir Das. ${ }^{8}$

Para confirmar y diferenciar Mycobacterium bovis de Mycobacterium tuberculosis, se desarrolló un ensayo de PCR en tiempo real basado en la presencia 0 ausencia de regiones de diferencia (RD1, Ext-RD9, RD4, RD9 y RD12) entre los genomas de los miembros de MTBC.

\section{Resultados}

Durante el periodo de estudio ingresaron 850 pacientes con sospecha de tuberculosis. En 441 casos se confirmó

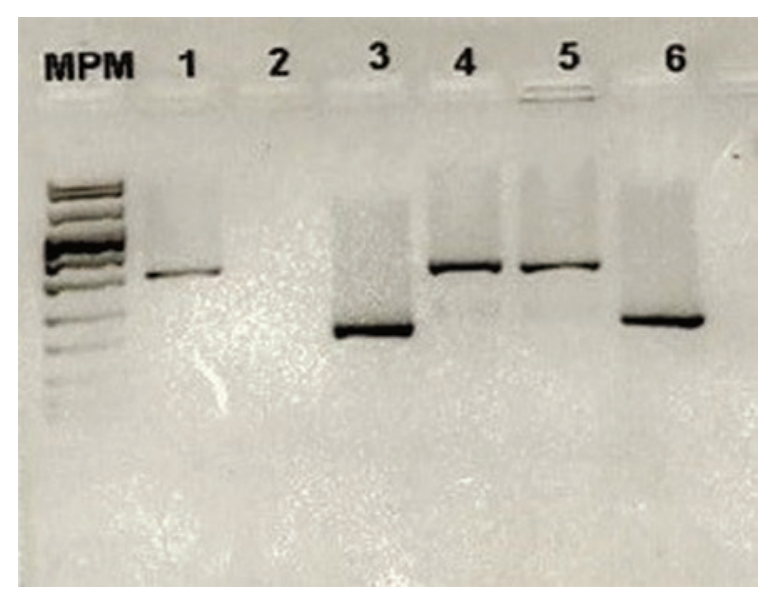

Figura 1. Diferenciación de Mycobacterium tuberculosis/Mycobacterium bovis mediante PCR punto final. Análisis del fragmento RD9 en un gel de agarosa a $2.5 \%$. MPM, marcador de tamaño molecular VIII (ROCHE); 1, cepa H37Rv de Mycobacterium tuberculosis; 2, control de reactivos de PCR; 3, Mycobacterium bovis BCG; 4 y 5, muestras 3209 y 1687, respectivamente, identificadas como Mycobacterium tuberculosis; 6, muestra 1916 identificada como Mycobacterium bovis.

Mycobacterium tuberculosis por cultivo Löwestein-Jensen y MGITI960 positivo, con un promedio de crecimiento de la cepa de 37 días. Se eliminaron 12 cultivos por contaminación; 70 \% (310) de los pacientes fue del sexo masculino y $30 \%$ (131) del femenino; la edad media fue de 41 años. El PPD fue positivo con una induración mayor a $10 \mathrm{~mm}$ en $25 \%$ de los pacientes.

En $30 \%$ de los casos se identificó síndrome de inmunodeficiencia adquirida (VIH/sida), en $25 \%$ diabetes mellitus y en $7 \%$, antecedentes de neoplasias en tratamiento con quimioterapia, cinco pacientes con uso crónico de corticosteroides.

La sintomatología que predominó fue fiebre (85\%), tos (82 \%), baja de peso (78\%), astenia y adinamia (72\%), disnea (60\%) y hemoptisis $(20 \%)$.

De los 441 casos con cultivo positivo, 250 (56.6\%) presentaron tuberculosis pulmonar, 65 (14.7\%) tuberculosis ganglionar, 39 (8.8\%) tuberculosis renal, 34 (7.7 \%) tuberculosis meníngea, 25 (5.6 \%) tuberculosis miliar, 14 (3.1\%) tuberculosis pleural, ocho (1.8\%) tuberculosis peritoneal, cuatro $(0.9 \%)$ tuberculosis ósea y dos $(0.4 \%)$ tuberculosis pericárdica. En todos los casos diagnosticados con tuberculosis se inició tratamiento con isoniazida, rifampicina, pirazinamida y etambutol, el cual fue estrictamente supervisado. La respuesta clínica fue evaluada durante un año después del diagnóstico. En la tipificación de las micobacterias se identificó Mycobacterium tuberculosis en $89.2 \%$.

En el presente estudio se registraron 48 (10.8\%) cepas que resultaron positivas a Mycobacterium bovis mediante la PCR de punto final de RD9 (Figura 1), todas positivas 


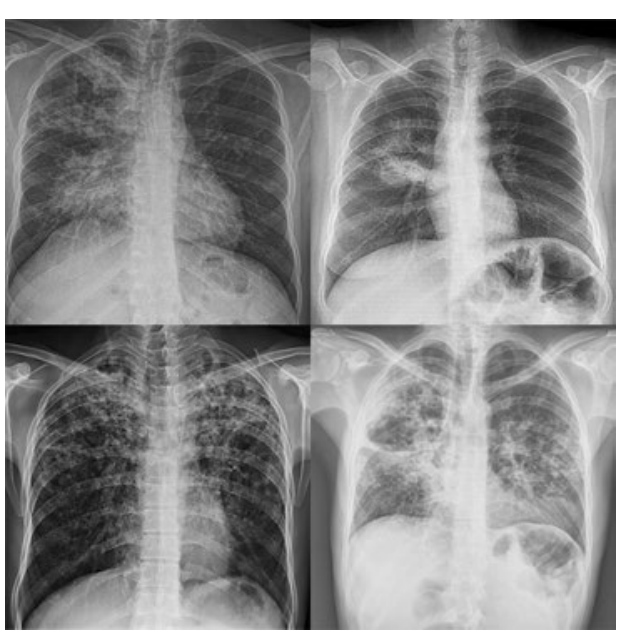

Figura 2. Radiografías de tórax en posición postero-anterior de pacientes con diagnóstico de tuberculosis pulmonar por Mycobacterium bovis en las que se observan múltiples lesiones en cavernas y con ocupación alveolar.

a Mycobacterium bovis con base en la presencia de RD1 y Ext-RD9 y la ausencia de marcadores RD4, RD9 y RD12, lo que representó $100 \%$ de concordancia entre los métodos. En este grupo se encontró $50 \%$ de hombres y $50 \%$ de mujeres. El PPD solo fue positivo en siete pacientes, con una induración promedio de $8 \mathrm{~mm}$.

Por su localización, 19 casos (39\%) fueron tuberculosis ganglionar, $10(21 \%)$ tuberculosis pulmonar, nueve (19\%) tuberculosis meníngea, siete (14\%) tuberculosis renal, dos (4\%) tuberculosis peritoneal y uno (2\%) tuberculosis pleural. El promedio de crecimiento en los cultivos fue de 31 días y la evolución de la enfermedad fue crónica, con un promedio de siete años.

\section{Discusión}

La infección por Mycobacterium bovis en el ganado bovino persiste en México y representa un problema de salud pública, a diferencia de los países de Europa occidental, declarados libres de tuberculosis bovina. ${ }^{9}$

En México, el manejo de la infección por Mycobacterium bovis es regulado por la Norma mexicana NOMZOO-031-1995. En México se desconoce la magnitud del problema de infección por Mycobacterium bovis en la población en general y en la población de riesgo, sin embargo, investigaciones aisladas han señalado variaciones de 2 a $40 \%{ }^{10}$ En México y Latinoamérica, así como en otros países en desarrollo, la prevalencia de enfermedad por Mycobacterium bovis no se encuentra documentada o es subestimada. ${ }^{11}$

La infección por Mycobacterium bovis tiene un espectro de la enfermedad más amplio, lo que provoca que la afección pulmonar progrese a etapas crónicas, como observamos en nuestro estudio: en los 10 pacientes con tuberculosis pulmonar se identificaron cavernas, bronquiectasias y fibrotórax, con una evolución de la enfermedad de nueve años (Figura 2). 12,13

La tuberculosis por Mycobacterium bovis se presenta sobre todo en un gran número de mamíferos domésticos y silvestres, en particular el ganado vacuno. Según la ubicación, en nuestra serie predominó la linfadenopatía cervical (39\%), lo que coincide con los informes de otras investigaciones La evolución del padecimiento fue de cuatro años y en cinco paciente fracasó el tratamiento de primera línea. ${ }^{13,14}$

En México se han realizado comparaciones de patrones de espoligotipos, los cuales coinciden con los observados en aislados de ganado, por lo que la tuberculosis en humanos causada por Mycobacterium bovis se deriva de las cepas que circula en el ganado vacuno. ${ }^{15}$ SB0120 es el tipo de circulación más común en varios continentes, mientras que SB0121 existe en Europa, África y América. ${ }^{16}$ La determinación del espoligotipo más común de Mycobacterium bovis es la clave para encontrar la fuente de infección y controlar y prevenir la enfermedad.

Los factores asociados con mayor prevalencia de la enfermedad son ser inmigrante, origen hispano, sexo femenino, uso de glucocorticoides, nivel socioeconómico bajo y presentaciones extrapulmonares, los cuales corroboramos en nuestro estudio: la edad media de los casos fue de 41 años, $45 \%$ de los casos provenía del medio rural y con bajos recursos económicos, ocho presentaban exposición ocupacional, $10 \mathrm{VIH} /$ sida con una cuenta de CD4 $<100$ células/mL. ${ }^{9,10} \mathrm{~A}$ diferencia de los resultados de otras investigaciones, no registramos diferencias en la frecuencia respecto al sexo de los pacientes. ${ }^{17}$

En los países emergentes, 10 a $20 \%$ de los casos de tuberculosis es causado por Mycobacterium bovis, sin embargo, este porcentaje puede ser subestimado porque la mayoría de los laboratorios no realizan rutinariamente cultivos de micobacterias y pocos cuentan con los sistemas para identificar Mycobacterium bovis. Existen pocos estudios que investiguen la diversidad genotípica y la resistencia a fármacos en Mycobacterium bovis a partir de infecciones en animales o humanos. ${ }^{18}$

En este estudio reportamos la presencia de Mycobacterium bovis en $10 \%$ de las cepas, resultado relevante debido a las repercusiones clínicas que representa. En ninguno de los 41 casos se sospechaba infección por Mycobacterium bovis. ${ }^{11}$

Las directrices actuales recomiendan un tratamiento más prolongado para la tuberculosis causada por organismos resistentes a pirazinamida como Mycobacterium 
bovis, con la finalidad de obtener cultivo negativo en esputo y tasa de curación más alta. En general se ha reportado resistencia a pirazinamida en $38 \%$ de los casos de grupos susceptibles, con mal pronóstico. ${ }^{19} \mathrm{En}$ nuestra serie, $20 \%$ de los pacientes presentó recaída al año de terminar el tratamiento, tres pacientes fallecieron a los cuatro meses posteriores al diagnóstico. ${ }^{20}$

En los cultivos identificados como Mycobacterium bovis se obtuvo un producto de amplificación de $206 \mathrm{pb}$ en el ensayo de PCR RD9, que correlacionó en $100 \%$ con los resultados del cultivo, pruebas bioquímicas y prueba moleculares. Por su facilidad, especificidad y bajo costo, el PCR RD9 resulta ser una herramienta de gran utilidad para el diagnóstico rutinario o la confirmación de Mycobacterium bovis en hospitales de tercer nivel, a diferencia de los espoligotipos, que si bien son una herramienta catalogada como poco laboriosa y que genera resultados reproducibles, están enfocados a estudios epidemiológicos y procesamiento de muestras a gran escala, además de su elevado costo. ${ }^{8}$

Actualmente es imprescindible contar con nuevos métodos de biología molecular en los hospitales de concentración de tercer nivel que demuestren su eficacia para identificar diferentes especies de micobacterias, como es el PCR multiplex, que permite diferenciar Mycobacterium bovis de Mycobacterium tuberculosis en esputo y en muestras extrapulmonares. ${ }^{20,21}$

Se necesitan estudios epidemiológicos moleculares en medicina y en campos veterinarios para definir la importancia de la tuberculosis zoonótica en la tuberculosis humana, conocer la ruta de transmisión, los factores de riesgo, los espoligotipo dominantes entre humanos y animales y comprender las relaciones filogeográficas de las cepas en México.

\section{Conclusión}

Actualmente no se piensa en Mycobacterium bovis como el agente etiológico de tuberculosis pulmonar y extrapulmonar, sin embargo, es importante realizar pruebas diagnósticas de biología molecular rápidas, sensibles y económicas, para estar en posibilidad de iniciar un tratamiento adecuado. Se requiere la colaboración entre los servicios veterinarios y de salud pública del país para disminuir la prevalencia de esta enfermedad.

\section{Agradecimientos}

A los miembros de los laboratorios de Diagnóstico y Tipificación Molecular y Genoma de Patógenos, por su participación en el presente estudio.

\section{Bibliografía}

1. Esteban J, Muñoz-Egea MC. mycobacterium bovis and other uncommon members of the Mycobacterium tuberculosis complex. Microbiol Spectr. 2016;4.

2. Olea-Popelka F, Muwonge A, Perera A, Dean AS, Mumford E, Erlacher-Vindel $\mathrm{E}$, et al. Zoonotic tuberculosis in human beings caused by Mycobacterium bovis a call for action. Lancet Infect Dis. 2017;17:1-5.

3. Scott C, Cavanaugh JS, Pratt R, Silk BJ, LoBue P, Moonan PK. Human tuberculosis caused by Mycobacterium bovis in the United States, 20062013. Clin Infect Dis. 2016;63:594-601.

4. Scott C, Cavanaugh JS, Silk BJ, Ershova J, Mazurek GH, LoBue PA, et al. Comparison of Sputum-culture conversion for Mycobacterium bovis and M. tuberculosis. Emerg Infect Dis. 2017;23:456-462.

5. Lombardi G, Botti I, Pacciarini ML, Boniotti MB, Roncarati G, Del Monte P. Five-year surveillance of human tuberculosis caused by Mycobacterium bovis in Bologn, Italy: an underestimated problem. Epidemiol Infect. 2017; 145:3035-3039.

6. Torres-González P, Cervera-Hernández ME, Martínez-Gamboa A, García-García L, Cruz-Hervert LP, Bobadilla-Del Valle M, et al. Human tuberculosis caused by Mycobacterium bovis: a retrospective comparison with Mycobacterium tuberculosis in a Mexican tertiary care centre, 20002015. BMC Infect Dis. 2016;16:657.

7. Kurbatova EV, Cavanaugh JS, Dalton T, Click ES, Cegielski JP. Epidemiology of pyrazinamide-resistant tuberculosis in the United States, 1999-2009. Clin Infect Dis. 2013;57:1081-1093.

8. Das S, Das SC, Verma R. Occurrence of RD9 region and 500 bp fragment among clinical isolates of Mycobacterium tuberculosis and Mycobacterium bovis. Microbiol Immunol. 2007;51:231-234.

9. Sandoval-Azuara SE, Muñiz-Salazar R, Perea-Jacobo R, Robbe-Austerman S, Perera-Ortiz A, López-Valencia G, et.al. Whole genome sequencing of mycobacterium bovis to obtain molecular fingerprints in human and cattle isolates from Baja California, Mexico. Int $\mathrm{J}$ Infect Dis. 2017;63:48-56.

10. Zaragoza-Bastida A, Rivero-Pérez N, Valladares-Carranza B, Isaac-Olivé K, Moreno-Pérez P, Sandoval-Trujillo H, et al. Molecular identification of mycobacterium species of public health and veterinary importance from cattle in the south state of México. Can J Infect Dis Med Microbiol. 2017;2017:6094587.

11. Müller B, Dürr S, Alonso S, Hattendorf J, Laisse CJ, Parsons SD, van Helden PD, Zinsstag J. Zoonotic mycobacterium bovis-induced tuberculosis in humans. Emerg Infect Dis. 2013;19:899-908.

12. Laniado-Laborín R, Muñiz-Salazar R, García-Ortiz RA, Vargas-Ojeda AC Villa-Rosas C, Oceguera-Palao L. Molecular characterization of Mycobacterium bovis isolates from patients with tuberculosis in Baja California, Mexico. Infect Genet Evol. 2014;27:1-5.

13. Siala M, Smaoui S, Taktak W, Hachicha S, Ghorbel A, Marouane C, et al. First-time detection and identification of the Mycobacterium tuberculosis complex members in extrapulmonary tuberculosis clinical samples in south Tunisia by a single tube tetraplex real-time PCR assay. PLoS Negl Trop Dis. 2017;5:e0005572.

14. Cicero R, Olivera H, Hernández SA, Ramírez CE, Escobar GA. Frequency of Mycobacterium bovis as an etiologic agent in extrapulmonary tuberculosis in HIV-positive and -negative Mexican patients. Eur J Clin Microbiol Infect Dis. 2009 May;28:455-460.

15. Perea-Razo CA, Rodríguez-Hernández E, Román-Ponce SI, Milián-Suazo F, Robbe-Austerman S, Stuber T, et al. Molecular epidemiology of cattle tuberculosis in Mexico through whole genome sequencing and spoligotyping. PLoS One. 2018;13:e0201981.

16. Ghavidel M, Mansury D, Nourian K, Ghazvini K. The most common spoligotype of Mycobacterium bovis isolated in the world and the recommended loci for VNTR typing; a systematic review. Microb Pathog. 2018;118:310-315

17. Silva MR, Rocha ADS, Araújo FR, Fonseca-Júnior AA, Alencar AP, Suffys PN, et al. Risk factors for human Mycobacterium bovis infections in an urban area of Brazil. Mem Inst Oswaldo Cruz. 2018:113:e170445.

18. Moghaddam R, Mosavari N, Mahalati AH. Molecular identification of Mycobacterium tuberculosis complex isolates from KermanshahProvince, Iran. Int J Mycobacteriol. 2016;5:S203.

19. Franco MMJ, Ribeiro MG, Pavan FR, Miyata M, Heinemann MB, De Souza AF, et al. Genotyping and rifampicin and isoniazid resistance in Mycobacterium bovis strains isolated from the lymph nodes of slaughtered cattle. Tuberculosis (Edinb). 2017;104:30-37.

20. Munir S, Mahmood N, Shahid S, Khan MI. Molecular detection of isoniazid, rifampin and ethambutol resistance to $\mathrm{m}$. tuberculosis and $\mathrm{M}$. bovis in multidrug resistant tuberculosis (MDR-TB) patients in Pakistan. Microb Pathog. 2017;110:262-274

21. Jabbar A, Khan J, Ullah A, Rehman H, Ali I. Detection of mycobacterium tuberculosis and Mycobacterium bovis from human sputum samples through multiplex PCR. Pak J Pharm Sci. 2015;28:1275-1280. 